Braz J Med Biol Res, January 2012, Volume 45(1) 13-19

doi: 10.1590/S0100-879X2011007500161

Hypoxic-ischemic injury decreases anxiety-like behavior in rats when associated with loss of tyrosine-hydroxylase immunoreactive neurons of the substantia nigra

Ming-Yan Hei, Ya-Li Luo, Xiao-Chun Zhang, Hong Liu, Ru Gao and Jing-Jiang Wu

The Brazilian Journal of Medical and Biological Research is partially financed by



Institutional Sponsors
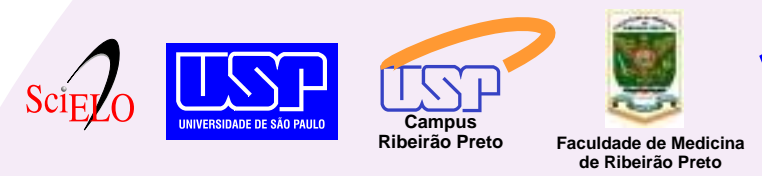

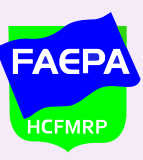

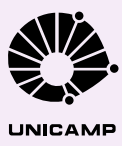

SHIMADZU

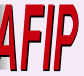

Associaçăo
Fundo de Incentivo
à Pesquisa



UNICAMP

lore High - Performance MS Orbitrap Technology analitica Thermo 


\title{
Hypoxic-ischemic injury decreases anxiety-like behavior in rats when associated with loss of tyrosine-hydroxylase immunoreactive neurons of the substantia nigra
}

\author{
Ming-Yan Hei, Ya-Li Luo, Xiao-Chun Zhang, Hong Liu, Ru Gao and Jing-Jiang Wu
}

Department of Pediatrics, the Third Xiangya Hospital, Central South University, Changsha, Hunan, China

\begin{abstract}
Neonatal Sprague-Dawley rats were randomly divided into normal control, mild hypoxia-ischemia (HI), and severe $\mathrm{HI}$ groups $(\mathrm{N}=10$ in each group at each time) on postnatal day 7 (P7) to study the effect of mild and severe HI on anxiety-like behavior and the expression of tyrosine hydroxylase (TH) in the substantia nigra (SN). The mild and severe HI groups were exposed to hypoxia $\left(8 \% \mathrm{O}_{2} / 92 \% \mathrm{~N}_{2}\right)$ for 90 and $150 \mathrm{~min}$, respectively. The elevated plus-maze (EPM) test was performed to assess anxiety-like behavior by measuring time spent in the open arms (OAT) and OAT\%, and immunohistochemistry was used to determine the expression of TH in the SN at P14, P21, and P28. OAT and OAT\% in the EPM were significantly increased in both the mild (1.88-, 1.99-, and 2.04-fold, and 1.94-, 1.51-, and 1.46-fold) and severe HI groups (1.69-, 1.68-, and 1.87-fold, and 1.83-, 1.43-, and 1.39-fold, respectively; $\mathrm{P}<0.05)$. The percent of TH-positive cells occupying the SN area was significantly and similarly decreased in both the mild $(17.7,40.2$, and $47.2 \%)$ and severe $\mathrm{HI}$ groups $(16.3,32.2$, and $43.8 \%$, respectively; P $<0.05)$. The decrease in the number of TH-positive cells in the SN and the level of protein expression were closely associated (Pearson correlation analysis: $r=0.991, \mathrm{P}=0.000$ in the mild $\mathrm{HI}$ group and $r=0.974, \mathrm{P}=0.000$ in the severe $\mathrm{HI}$ group) with the impaired anxiety-like behaviors. We conclude that neonatal $\mathrm{HI}$ results in decreased anxiety-like behavior during the juvenile period of Sprague-Dawley rats, which is associated with the decreased activity of TH in the SN. The impairment of anxiety and the expression of TH are not likely to be dependent on the severity of HI.
\end{abstract}

Key words: Anxiety; Tyrosine hydroxylase; Substantia nigra; Hypoxia-ischemia

\section{Introduction}

Perinatal asphyxia is one of the major causes of neonatal morbidity and mortality. The incidence of perinatal asphyxia ranges from 1 to 8 per 1000 live births in the US (1); however, no national data are currently available in China. During the early stage of life, the brain is particularly vulnerable to hypoxic-ischemic $(\mathrm{HI})$ insults and the outcome varies from mild behavioral dysfunctions to severe mental retardation and motor disturbances. Mild exposure to hypoxia in the early stage of brain development results in subtle behavioral abnormalities, including attention deficit combined with anxiety, in spite of the lack of detectable brain damage in adult life $(2,3)$. Early brain damage can influence development by changing the expression of genetic traits that interact with other characteristics of children. On this basis, an early hypoxic event resulting in neurological dysfunction can influence development directly and indirectly, leading to different developmental pathways in children.

Perinatal $\mathrm{HI}$-induced behavioral disturbances have been grouped into two categories, hyperactivity and learning/ memory deficits (4). Evidence has shown that locomotor activity disorders are associated with an abnormal level of dopamine content in the rat brain with simultaneous substantia nigra (SN) injury (5). Anxiety-like behavior is associated with the levels of dopamine and its metabolites in the mouse brain following ischemia (6). Dopamine is regulated by the activity of tyrosine hydroxylase $(\mathrm{TH})$ and the expression of $\mathrm{TH}$ reflects the function of dopaminergic neurons (7). Damage to dopaminergic neurons can be visualized directly by TH immunohistochemistry and measured by the level of $\mathrm{TH}$ protein expression (8).

Correspondence: Ming-Yan Hei, Department of Pediatrics, the Third Xiangya Hospital, Central South University, Hunan, Changsha, 410013 China. E-mail: heiming_yan@yahoo.com.cn

Received May 22, 2011. Accepted November 28, 2011. Available online December 9, 2011. Published January 16, 2012. 
Neonatal $\mathrm{HI}$ encephalopathy is brain damage caused by the combination of hypoxia and ischemia, and it is mostly attributed to perinatal asphyxia (9). It has been shown that less anxiety-like behavior is observed in animals subjected to $\mathrm{HI}(10)$. Current animal studies regarding ischemia or hypoxia are complex, and even conflicting findings of anxiety-like behaviors are obtained. It was reported that increased anxiety (11) or no changes in anxiety (12) was found in animals subjected to global ischemia, and increased anxiety (2) or no changes in anxiety (13) has been found after cerebral hypoxia $(14,15)$.

Clinical studies have reported an elevated rate of anxiety in children with mild and moderate neonatal encephalopathy (NE) following perinatal asphyxia (16). Furthermore, more problems were found to be related to anxiety at school age in a mixed group of children with NE compared to a control group (17), indicating that the perinatal asphyxia insult indeed has a developmental impact that lasts at least until middle school age. Clinical data have shown that behavior impairment was more often present in children with moderate to severe NE, but not in children with mild NE following asphyxia (18). Therefore, it is necessary to clarify the effect of the severity of $\mathrm{HI}$ on anxiety and related mechanisms.

In the present study, we used a Rice-Vannucci HIBD rat model (19) with different hypoxic exposure to determine whether a mild or severe $\mathrm{HI}$ insult caused different anxietylike behaviors, and whether the change of behavior was related to $\mathrm{TH}$ activity in the $\mathrm{SN}$.

\section{Material and Methods}

\section{Animal preparation}

All animal procedures were approved by the Animal Ethics Committee of Central South University. SpragueDawley (SD) rats on postnatal day 7 (P7; both genders, body weight 11-16 g) were randomly divided into normal control, severe $\mathrm{HI}$, and mild $\mathrm{HI}$ groups. In the $\mathrm{HI}$ groups, pups were lightly anesthetized with isoflurane (4\% induction, $1.5 \%$ maintenance). The Rice-Vannucci method was used to induce $\mathrm{HI}$ brain damage (19). Ligation of the right common carotid artery was performed under a surgical microscope. After the wound was sutured, animals were placed on a warm heating pad $\left(34^{\circ} \mathrm{C}\right)$ for recovery from anesthesia for $30 \mathrm{~min}$. In the mild and severe $\mathrm{HI}$ groups, animals were exposed to hypoxia $\left(8 \% \mathrm{O}_{2} / 92 \% \mathrm{~N}_{2}\right)$ for 90 and 150 min (20), respectively.

\section{Elevated plus-maze (EPM) test}

The EPM test was performed on P14, P21, and P28 ( $N=10$ in each group at each time). The EPM test is widely used to observe the behaviors, which are relevant for understanding anxiety in animal studies $(21,22)$. The EPM apparatus consists of two open arms (50 x $10 \times 1$ $\mathrm{cm})$ and two enclosed arms $(50 \times 10 \times 40 \mathrm{~cm})$ originating from a common central platform $(10 \times 10 \mathrm{~cm})$ to form a plus shape. The entire apparatus was elevated to a height of $50 \mathrm{~cm}$ above the floor. A video camera and illumination lamps were mounted on the ceiling. The anxiety-related behaviors of each animal were recorded for a period of 5 min by a VCR-recording system on P19. At the beginning of the test, the rat was placed on the central platform with its head facing an open arm. The arm entry was defined as all four paws into an open or closed arm. The total time each animal spent in various sections of the maze (open arms, center, or enclosed arms) was recorded. The results are reported as open arm time (OAT), percent of OAT (OAT\%, time spent in open arms divided by the sum of time spent in both closed and open arms).

\section{TH immunohistochemistry (IHC)}

At P14, P21 and P28 ( $N=10$ in each group at each time), rat pups were anesthetized with $4 \%$ isoflurane and sacrificed by transcardial perfusion with physiological saline followed by $4 \%$ paraformaldehyde. After being cryoprotected with $30 \%$ sucrose in phosphate-buffered solution (PBS), $\mathrm{pH}$ 7.4 , the brain was cryosectioned into $10-\mu \mathrm{m}$ thick coronal sections and collected onto Chrome-Album-coated slides and air-dried. The coronal sections of the midbrain were cut at a level $1 / 3$ rostrally from the lambda to the bregma. The sections were incubated with monoclonal mouse anti-rat antibody to $\mathrm{TH}\left(1: 200\right.$, Chemicon, USA) at $4^{\circ} \mathrm{C}$ overnight, washed in PBS, and then incubated with biotinylated goat anti-mouse secondary antibody (1:400, Vector, USA) at room temperature for $2 \mathrm{~h}$, followed by incubation by the avidin-biotin complex method (ABC elite kit, Vector, USA). Finally, the sections were visualized with 3,3V-diaminobenzidine tetrachloride (DAB, Sigma, USA). A Nikon Eclipse 80i microscope was used for observation, and a Nikon DS digital camera equipped with the NIS-Elements AR Version 3.0 software was used to photograph and study the images. The data are reported as percent of TH-positive cells occupying the area in the $\mathrm{SN}$, which was calculated as positive-staining area divided by the total area of the section. The result was the average of the three sections in each brain. We took the first section in every 3 consecutive sections with an interval of $30 \mu \mathrm{m}$.

\section{Statistical analysis}

The SPSS 16.0 software was used to analyze the data (supplied by Department of Medical Statistics, Institute of Public Health, Central South University, China). To avoid bias, all histological and behavioral analyses were carried out by an operator who did not know from which hypoxia treatment group the tissue or video was obtained. Data are reported as means $\pm S D$ and were analyzed by oneway ANOVA followed by the Student-Newman-Keuls test. Results with a $\mathrm{P}<0.05$ were considered to be statistically significant. Pearson correlation analysis was used to study the correlation between the change of OAT in the EPM and the percent of $\mathrm{TH}$-positive cells in IHC. 


\section{Results}

\section{Mild and severe HI significantly increased OAT and OAT\% detected by the EPM test}

The EPM test was used to assess the level of anxiety. It is known that a longer OAT and a higher OAT\% indicate a lower level of anxiety (23). In the present study, longer OAT and higher OAT\% (Table 1) were observed in both the mild and severe $\mathrm{HI}$ groups compared to the normal control group at all times examined. We found that time spent in the open arms by rats in the mild HI group was significantly increased by 1.88-, 1.99-, and 2.04-fold ( $P<0.05)$, OAT\% was also increased by 1.94-, 1.51-, and 1.46-fold ( $P<0.05)$, when compared to control at P14, P21, and P28. Similarly, in the severe HI group, OAT was significantly increased by 1.69-, 1.68-, and 1.87-fold $(P<0.05)$, and OAT\% was significantly increased by $1.83-$,
1.43-, and 1.39-fold ( $P<0.05)$. Although both OAT and OAT\% were slightly increased in the mild $\mathrm{HI}$ group compared to the severe $\mathrm{HI}$ group at all times examined, statistical analysis demonstrated no significant difference between these two groups $(P>0.05)$.

\section{$\mathrm{HI}$ induced down-regulation of TH expression}

TH immunohistochemistry has been used to detect the damage to dopaminergic neurons (8), which is found to be associated with anxiety-like behavior (6). To examine whether $\mathrm{HI}$ induced a change in $\mathrm{TH}$ expression, we determined its expression in the $\mathrm{SN}$ after $\mathrm{HI}$ at different postnatal times. An abundant number of $\mathrm{TH}$-positive cells in the $\mathrm{SN}$ were found in the normal control group, while the number was significantly decreased in both the mild and severe HI groups (Figure 1). This change can also be observed under a higher magnifi-

Table 1. Effect of hypoxia-ischemia (HI) on anxiety-like behavior measured by the elevated plus-maze test.

\begin{tabular}{lccccccc}
\hline & \multicolumn{3}{c}{ OAT $(\mathrm{s})$} & & \multicolumn{3}{c}{ OAT\% } \\
\cline { 2 - 3 } \cline { 6 - 7 } & P14 & P21 & P28 & & P14 & P21 & P28 \\
\hline Control & $28.33 \pm 9.21$ & $48.33 \pm 33.53$ & $45.27 \pm 22.22$ & & $21.63 \pm 10.61$ & $31.50 \pm 9.20$ & $32.06 \pm 16.74$ \\
Severe HI & $48.00 \pm 10.00^{*}$ & $81.33 \pm 10.51^{*}$ & $84.97 \pm 13.99^{*}$ & & $39.66 \pm 12.69^{*}$ & $45.17 \pm 7.61^{*}$ & $44.75 \pm 17.04^{*}$ \\
Mild HI & $53.33 \pm 11.04^{*}$ & $96.33 \pm 25.31^{*}$ & $92.33 \pm 12.44^{*}$ & & $42.07 \pm 13.69^{*}$ & $47.67 \pm 8.96^{*}$ & $46.81 \pm 11.42^{*}$ \\
\hline
\end{tabular}

Data are reported as means \pm SD for $N=10$ Sprague-Dawley rats in each group at postnatal days (P) 14, 21, and 28. OAT = open arm time. ${ }^{*} \mathrm{P}<0.05$ for the severe or mild $\mathrm{HI}$ group compared to control (one-way ANOVA followed by the Student-Newman-Keuls test).

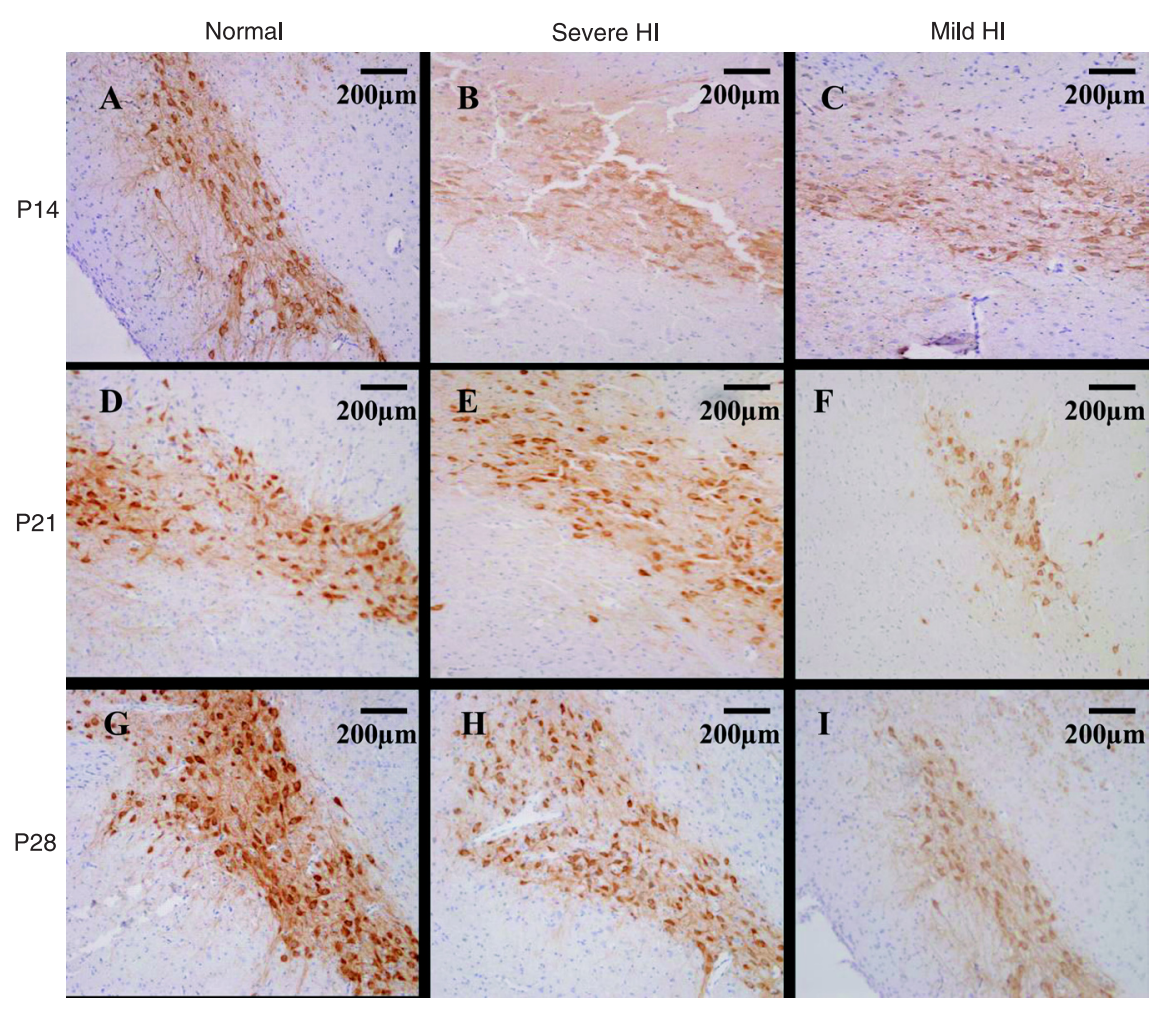

Figure 1. Immunohistochemical staining of tyrosine hydroxylase (TH) in the substantia nigra (SN) of rats subjected to hypoxia-ischemia $(\mathrm{HI})$ on postnatal day $(\mathrm{P}) 7$ at P14, P21, P28 $(\mathrm{N}=10)$. There were abundant $\mathrm{TH}$-positive cells (brown color) in the $\mathrm{SN}$ of the normal control group at all times $(A, D, G)$. The number and intensity of $\mathrm{TH}$-positive cells significantly decreased in both severe $(B, E, H)$ and mild $(C, F, I) \mathrm{HI}$ groups. Magnification: 100X. 




Figure 2. Higher magnification of the immunohistochemical $\mathrm{TH}$ staining in Figure 1. The rats were subjected to hypoxia-ischemia $(\mathrm{HI})$ on postnatal day $(\mathrm{P}) 7$ and data are reported for P14, P21, and P28 $(\mathrm{N}=10)$. The number of $\mathrm{TH}$-positive cells in the normal control group at all times $(A$, $D, G)$ was higher than that in both the severe $(B, E, H)$ and mild $(C, F, I) \mathrm{HI}$ groups. TH-positive cells were morphologically normal neurons with positive cytoplasm staining (brown color). Magnification: 400X. TH = tyrosine hydroxylase; $\mathrm{SN}=$ substantia nigra.

cation microscope (Figure 2), in which the positive staining was mainly distributed in the cytoplasm. TH-positive cells were morphologically normal with an intact cell membrane and normal nuclei. Based on their morphology, these cells were neurons. The percent of $\mathrm{TH}$-positive cells occupying the area in the $\mathrm{SN}$ in the mild $\mathrm{HI}$ group was markedly decreased by $17.7,40.2$, and $47.2 \%(P<0.05)$, respectively, when compared to control at P14, P21, and P28. Similarly, in the severe $\mathrm{HI}$ group, the percent was also decreased by $16.3,32.2$, and $43.8 \%$, respectively $(P<0.05)$. There was no significant difference $(P>0.05)$ between the mild and severe $\mathrm{HI}$ groups (Table 2 ).

\section{Correlation between the change of OAT and percent TH expression}

To examine whether a correlation existed between the change of OAT and TH expression in IHC, Pearson correlation analysis was performed. The results showed that the coefficient index of the change of OAT in the EPM test and the percent of the area occupied by $\mathrm{TH}$-positive cells in $\mathrm{IHC}$ was $r=0.991(\mathrm{P}=0.000)$ in the mild $\mathrm{HI}$ group and $r$ $=0.974(\mathrm{P}=0.000)$ in the severe $\mathrm{HI}$ group, indicating that the down-regulation of $\mathrm{TH}$ expression was associated with the decreased anxiety-like behaviors.

\section{Discussion}

The major findings of the present study were that both mild and severe $\mathrm{HI}$ insults during the neonatal period result in a decreased anxiety-like behavior during the juvenile pe-
Table 2. Percent of tyrosine hydroxylase-positive cells occupying the area of the substantia nigra after hypoxia-ischemia $(\mathrm{HI})$ was applied on day P7.

\begin{tabular}{lccc}
\hline & P14 & P21 & P28 \\
\hline Normal & $4.58 \pm 1.03$ & $5.69 \pm 1.40$ & $5.78 \pm 1.68$ \\
Severe HI & $3.83 \pm 0.82^{*}$ & $3.86 \pm 0.62^{*}$ & $3.25 \pm 1.02^{*}$ \\
Mild HI & $3.77 \pm 0.91^{*}$ & $3.40 \pm 0.95^{*}$ & $3.05 \pm 0.84^{*}$ \\
\hline
\end{tabular}

Data are reported as means \pm SD for $N=10$ Sprague-Dawley rats in each group at postnatal days $(P) 14,21$, and 28 . ${ }^{*} P<0.05$ for the severe or mild $\mathrm{HI}$ group compared to control (one-way ANOVA followed by the Student-Newman-Keuls test).

riod in SD rats. The decrease of the number of $\mathrm{TH}$-positive cells in the $\mathrm{SN}$ and of the level of protein expression were associated with the decreased anxiety-like behaviors. However, neither the impairment of anxiety nor the expression of TH were dependent on the severity of $\mathrm{HI}$. These findings provide further information on the mechanism underlying hypoxic-ischemic brain injury and the anxiety-like behavior abnormality caused by perinatal $\mathrm{HI}$.

The majority of studies concerning perinatal asphyxia focus on the detection of major developmental abnormalities at a very young age (24) or biopathophysiological changes within several days after birth $(25,26)$, while behavioral changes such as anxiety during the juvenile period are one of the important focuses in long-term neurological outcomes after perinatal $\mathrm{HI}$ insult (27). The present study 
was novel because it targeted the effect of a neonatal $\mathrm{HI}$ insult on anxiety during the period from 1 week after the $\mathrm{HI}$ insult to 3 weeks after $\mathrm{HI}$, a time that corresponds to the human juvenile period. For SD rats, P28 is the weaning time when male and female rats mate with each other. P21 has been used as a time point for studying the neurobehavioral performance of juvenile SD rats $(14,28)$. In addition, findings for a series of times in this study (P14, P21, and P28) were considered to be the dynamic changes during the juvenile period.

Knyazev et al. (29) wrote that "anxiety is seen as being most often generated by concurrent and equivalent activation of fear (or frustration) and reward systems. Therefore, anxiety should be most evident in a situation of uncertainty when chances of winning and losing are about equal. If uncertainty disappears, anxiety should give way to satisfaction or joy (if winning) or some negative emotions ranging from sadness to fear". Exposure to novelty has been shown to induce anxiety responses in a variety of behavioral paradigms. Most current models of anxiety consist of exposure of animals to novelty in an anxiogenic environment (30). EPM is a widely used animal test of anxiety based on the curiosity of rats and their familiarization with the environment induced by previous experiences in the EPM, which could lead to a reduction in the approach/ avoidance of conflict (31). Results from the present EPM test showed that the $\mathrm{HI}$ insult increased the number of OAT and OAT\%. The behaviors measured are relevant for understanding anxiety. A reduction in $\mathrm{HI}$-induced anxiety-like behavior has also been reported in other animal models of perinatal asphyxia $(14,15)$. In the present study, there was no significant difference in OAT or OAT\% between the mild and severe $\mathrm{HI}$ groups, indicating the possibility that the duration of hypoxic exposure or the severity of the $\mathrm{HI}$ insult might not contribute the most to the impairment of anxiety-like performance. On the other hand, Fan et al. (28) reported that neonatal $\mathrm{HI}$ resulted in not only persistent white matter injury, but also in a higher proportion of open arm entry in the EPM test. In addition, they found that both brain damage and behavioral impairment were dependent on the duration of hypoxic exposure. The possible explanations for the differences are: 1) P4 rat pups were used in Fan's study, whereas P7 rat pups were used in the present study, and 2) the $\mathrm{HI}$ insult was induced by bilateral carotid artery occlusion followed by exposure to hypoxia in Fan's study, whereas the $\mathrm{HI}$ insult was induced by unilateral ligation of the common carotid artery followed by exposure to hypoxia in the present study.

Basal ganglia injury is common in hypoxic-ischemic brain damage (HIBD). Clinical autopsy data have proven that up to $43 \%$ of $\mathrm{HI}$ exposed neonates have basal ganglia injury (32). It has been reported that behavioral problems are associated with an abnormal level of dopamine content in the rat brain concomitant with SN injury (5). It is well known that dopamine is one of the cholinergic neurotransmitters regulated by $\mathrm{TH}$. In the present study, in both the mild and severe $\mathrm{HI}$ groups, the expression of $\mathrm{TH}$ in the $\mathrm{SN}$ was reduced from P14 to P28, indicating a persistent damage of dopaminergic neurons after $\mathrm{HI}$. This finding was consistent with previous studies showing that $\mathrm{HI}$ significantly reduced the number of TH-positive cells in SD rats on P21 (28) and in Wistar rats on P9 (33) after a neonatal $\mathrm{HI}$ insult, and this reduction during the neonatal period was reported to result in a decreased adult number of SN neurons (34). Moreover, in the present study, Pearson correlation analysis indicated that the down-regulation of TH expression was associated with the decreased anxiety-like behaviors. The expression of $\mathrm{TH}$ represents the function of dopaminergic neurons, and it has been commonly used to study dopamine-related neurodegenerative diseases such as Parkinson disease (7). The altered $\mathrm{TH}$ activity in the $\mathrm{SN}$ of juvenile rats in the current study may be part of the neural mechanisms contributing to the reduced anxiety-like behavior induced by neonatal HI. However, the present study showed neither any timedependent change of $\mathrm{TH}$ activity during the juvenile period of SD rat, nor any difference in the change of TH activity between the mild and severe HI groups. This is inconsistent with the findings reported by Burke et al. (34), who showed that both the reduction in the number of $\mathrm{TH}$-positive neurons in the $\mathrm{SN}$ and the extent of hyperactivity in the juvenile rat (P21) following HI depended on hypoxia duration. One of the possible explanations is that the duration of hypoxia differed between the present study and the Burke et al. study (34). The HI model in the Burke et al. study consisted of applying unilateral carotid ligation followed by 3- to 4-h exposure to $8 \% \mathrm{O}_{2}$ to $\mathrm{P} 7$ rats, while the $\mathrm{HI}$ model in the present study consisted of applying unilateral carotid ligation followed by $90-(1.5 \mathrm{~h})$ or $150-\min (2.5 \mathrm{~h})$ exposure to $8 \% \mathrm{O}_{2}$ in $\mathrm{P} 7$ rats. Further studies are needed to clarify whether more precise control of the duration of hypoxia would affect the changes of $\mathrm{TH}$-positive neurons in the $\mathrm{SN}$ and the hyperactivity in the juvenile rat after $\mathrm{HI}$.

\section{Acknowledgments}

We thank Department of Anatomy, Institute of Medicine of Central South University for supplying the apparatus for the EPM test. We thank Medjaden Bioscience Limited for assisting with the preparation of this manuscript. Research supported by Hunan Science \& Technological Research Foundation (\#2008FJ3157) and College Innovation and Creative Education Program of Central South University (\#YD0919). 


\section{References}

1. American College of Obstetrics and Gynecology: Task force on neonatal encephalopathy. Neonatal encephalopathy and cerebral palsy: Defining the pathogenesis and pathophysiology. Washington: American College of Obstetrics and Gynecology; 2003.

2. Grojean S, Schroeder H, Pourie G, Charriaut-Marlangue C, Koziel V, Desor D, et al. Histopathological alterations and functional brain deficits after transient hypoxia in the newborn rat pup: a long term follow-up. Neurobiol Dis 2003; 14 : 265-278.

3. Casolini P, Zuena AR, Cinque C, Matteucci P, Alema GS, Adriani $\mathrm{W}$, et al. Sub-neurotoxic neonatal anoxia induces subtle behavioural changes and specific abnormalities in brain group-I metabotropic glutamate receptors in rats. $J$ Neurochem 2005; 95: 137-145.

4. Nyakas C, Buwalda B, Luiten PG. Hypoxia and brain development. Prog Neurobiol 1996; 49: 1-51.

5. Bakos J, Duncko R, Makatsori A, Pirnik Z, Kiss A, Jezova $D$. Prenatal immune challenge affects growth, behavior, and brain dopamine in offspring. Ann N Y Acad Sci 2004; 1018: 281-287.

6. Winter B, Juckel G, Viktorov I, Katchanov J, Gietz A, Sohr $\mathrm{R}$, et al. Anxious and hyperactive phenotype following brief ischemic episodes in mice. Biol Psychiatry 2005; 57: 11661175.

7. Shi J, Yu WJ, Sun GY, Zhu SW. Organotypie brain slice triple culture of neocortex-striatum-substa-nigra of neonatal SD rats. Progr Anat Sci 2006; 12: 18-20.

8. Testa CM, Sherer TB, Greenamyre JT. Rotenone induces oxidative stress and dopaminergic neuron damage in organotypic substantia nigra cultures. Brain Res Mol Brain Res 2005; 134: 109-118.

9. Armstrong-Wells J, Bernard TJ, Boada R, Manco-Johnson $M$. Neurocognitive outcomes following neonatal encephalopathy. NeuroRehabilitation 2010; 26: 27-33.

10. Yan XB, Wang SS, Hou HL, Ji R, Zhou JN. Lithium improves the behavioral disorder in rats subjected to transient global cerebral ischemia. Behav Brain Res 2007; 177: 282-289.

11. Dhooper A, Young C, Reid KH. Ischemia-induced anxiety following cardiac arrest in the rat. Behav Brain Res 1997; 84: $57-62$.

12. Roberge MC, Hotte-Bernard J, Messier C, Plamondon $\mathrm{H}$. Food restriction attenuates ischemia-induced spatial learning and memory deficits despite extensive CA1 ischemic injury. Behav Brain Res 2008; 187: 123-132.

13. Buwalda B, Nyakas C, Vosselman HJ, Luiten PG. Effects of early postnatal anoxia on adult learning and emotion in rats. Behav Brain Res 1995; 67: 85-90.

14. Fan LW, Lin S, Pang Y, Rhodes PG, Cai Z. Minocycline attenuates hypoxia-ischemia-induced neurological dysfunction and brain injury in the juvenile rat. Eur J Neurosci 2006; 24: 341-350.

15. Caputa M, Rogalska J, Wentowska K, Nowakowska A. Perinatal asphyxia, hyperthermia and hyperferremia as factors inducing behavioural disturbances in adulthood: a rat model. Behav Brain Res 2005; 163: 246-256.

16. van Handel M, Swaab H, de Vries LS, Jongmans MJ. Behavioral outcome in children with a history of neonatal encephalopathy following perinatal asphyxia. J Pediatr Psychol
2010; 35: 286-295.

17. Moster D, Lie RT, Markestad T. Joint association of Apgar scores and early neonatal symptoms with minor disabilities at school age. Arch Dis Child Fetal Neonatal Ed 2002; 86: F16-F21.

18. Marlow N, Rose AS, Rands CE, Draper ES. Neuropsychological and educational problems at school age associated with neonatal encephalopathy. Arch Dis Child Fetal Neonatal Ed 2005; 90: F380-F387.

19. Rice JE III, Vannucci RC, Brierley JB. The influence of immaturity on hypoxic-ischemic brain damage in the rat. Ann Neurol 1981; 9: 131-141.

20. Matchett GA, Fathali N, Hasegawa $Y$, Jadhav V, Ostrowski $\mathrm{RP}$, Martin RD, et al. Hydrogen gas is ineffective in moderate and severe neonatal hypoxia-ischemia rat models. Brain Res 2009; 1259: 90-97.

21. Vargas KM, Da Cunha $C$, Andreatini R. Amphetamine and pentylenetetrazole given post-trial 1 enhance one-trial tolerance to the anxiolytic effect of diazepam in the elevated plusmaze in mice. Prog Neuropsychopharmacol Biol Psychiatry 2006; 30: 1394-1402.

22. Stock H, Foradori C, Ford K, Wilson MA. A lack of tolerance to the anxiolytic effects of diazepam on the plus-maze: comparison of male and female rats. Psychopharmacology 2000; 147: 362-370.

23. Pellow S, Chopin P, File SE, Briley M. Validation of open:closed arm entries in an elevated plus-maze as a measure of anxiety in the rat. $J$ Neurosci Methods 1985; 14 : 149-167.

24. van Handel M, Swaab H, de Vries LS, Jongmans MJ. Longterm cognitive and behavioral consequences of neonatal encephalopathy following perinatal asphyxia: a review. Eur J Pediatr 2007; 166: 645-654.

25. Calvert JW, Zhou C, Nanda A, Zhang JH. Effect of hyperbaric oxygen on apoptosis in neonatal hypoxia-ischemia rat model. J Appl Physiol 2003; 95: 2072-2080.

26. Sung DK, Chang YS, Kang S, Song HY, Park WS, Lee BH. Comparative evaluation of hypoxic-ischemic brain injury by flow cytometric analysis of mitochondrial membrane potential with JC-1 in neonatal rats. J Neurosci Methods 2010; 193: 232-238.

27. Katz LY, Fotti SA, Postl L. Cognitive-behavioral therapy and dialectical behavior therapy; adaptations required to treat adolescents. Psychiatr Clin North Am 2009; 32: 95-109.

28. Fan LW, Lin S, Pang Y, Lei M, Zhang F, Rhodes PG, et al. Hypoxia-ischemia induced neurological dysfunction and brain injury in the neonatal rat. Behav Brain Res 2005; 165: 80-90.

29. Knyazev GG, Savostyanov AN, Levin EA. Uncertainty, anxiety, and brain oscillations. Neurosci Lett 2005; 387: 121-125.

30. Ennaceur A, Michalikova S, Chazot PL. Models of anxiety: responses of rats to novelty in an open space and an enclosed space. Behav Brain Res 2006; 171: 26-49.

31. Carobrez AP, Bertoglio LJ. Ethological and temporal analyses of anxiety-like behavior: the elevated plus-maze model 20 years on. Neurosci Biobehav Rev 2005; 29: 1193-1205.

32. Bai XL, Meng SZ, Gao DN, Han YK. Pathological features, expression changes of dopamine transporter and dopamine 
D2 receptor in neonatal hypoxic-ischemic basal ganglia injury. Chin J Neonatol 2001; 16: 255-258.

33. Oo TF, Henchcliffe C, Burke RE. Apoptosis in substantia nigra following developmental hypoxic-ischemic injury. Neuroscience 1995; 69: 893-901.
34. Burke RE, Macaya A, DeVivo D, Kenyon N, Janec EM. Neonatal hypoxic-ischemic or excitotoxic striatal injury results in a decreased adult number of substantia nigra neurons. Neuroscience 1992; 50: 559-569. 